\title{
COMPARAÇÃO DA EFICIÊNCIA DAS COLORAÇÕES DE ZIEHL- NEELSEN MODIFICADO E SAFRANINA MODIFICADA NA DETECÇÃO DE OOCISTOS DE Cryptosporidium spp. (EUCOCCIDIORIDA, CRYPTOSPORIDIIDAE) A PARTIR DE AMOSTRAS FECAIS DE BEZERROS DE 0 A 3 MESES
}

\author{
COMPARISON OF EFFICIENCY OF STAINING WITH ZIEHL-NEELSEN \\ MODIFIED AND SAFRANIN MODIFIED IN DETECTION OF Cryptosporidium \\ spp. (EUCOCCIDIORIDA, CRYPTOSPORIDIIDAE) FROM FECAL SAMPLES \\ OF CALVES FROM O AND 3 MONTHS
}

\author{
Renata Dias Rodrigues ${ }^{1 *}$ \\ Lara Reis Gomes ${ }^{1}$ \\ Rafael Rocha de Souza ${ }^{1}$ \\ Fernando Cristino Barbosa ${ }^{1}$ \\ ${ }^{1}$ Universidade Federal de Uberlândia, Uberlândia, MG, Brasil. \\ *Autora para correspondência - renatavetufu@gmail.com
}

\section{Resumo}

A criptosporidiose bovina é causada principalmente por quatro espécies distintas: Cryptosporidium parvum, Cryptosporidium bovis, Cryptosporidium ryanae e Cryptosporidium andersoni. A espécie Cryptosporidium parvum (Ordem: Eucoccidiorida, Família: Cryptosporidiidae) é considerada de alto potencial zoonótico, podendo infectar humanos por intermédio da eliminação de oocistos tanto pelos bovinos quanto pelo próprio humano. O objetivo desta pesquisa foi verificar a ocorrência de oocistos de Cryptosporidium spp. em amostras fecais de bezerros ( 75 machos e 77 fêmeas), tendo sido coletadas 152 amostras de fezes de animais do nascimento até os três meses de idade. O material foi submetido às técnicas de coloração de Ziehl-Neelsen modificado e Safranina modificada, as lâminas foram observadas em toda sua extensão ao microscópio óptico para a verificação da presença de oocistos desta enteroparasitose. Os resultados demonstraram 17,1\% (26/152) de positividade no total das amostras examinadas e a análise estatística revelou não haver diferença entre o sexo e as técnicas de coloração utilizadas neste estudo. Conclui-se que a infecção por Cryptosporidium spp. esta presente nas propriedades avaliadas, porém são necessários mais estudos para que o risco de infecção seja mensurado adequadamente e medidas profiláticas implementadas.

Palavras-chave: enteroparasitose; ruminante; zoonose.

\begin{abstract}
Bovine cryptosporidiosis is caused by four differents species: Cryptosporidium parvum, Cryptosporidium bovis, Cryptosporidium andersoni and Cryptosporidium ryanae. The species Cryptosporidium parvum (Order: Eucoccidiorida, Family: Cryptosporidiidae) is considered of high zoonotic potential and it can infect humans through the elimination of oocysts by both cattle and by humans. The objective of this research was to detect oocysts of the genus Cryptosporidium spp. in fecal contents of calves ( 75 males and 77 females). We collected 152 stool samples from animals aged between 0 day and 3 months. The material was subjected to modified Ziehl-Neelsen and modified Safranin techniques, the slides were observed in its entire length by optical microscopy to verify the
\end{abstract}


presence of oocysts of this parasitic infections. The results showed $17.1 \%(26 / 152)$ positivity in the samples examined, and the statistical analysis showed no difference between sex and the staining techniques used in this study. We concluded the infection by Cryptosporidium spp. is present in the evaluated properties, but more studies are needed, so that the risk of infection is measured properly and prophylactic measures are implemented.

Keywords: enteroparasitosis; ruminant; zoonosis.

Enviado em: 28 julho 2014

Aceito em: 19 novembro 2015

\section{Introdução}

A Criptosporidiose é uma doença causada por coccídeos da família Cryptosporidiidae; o gênero Cryptosporidium compreende 25 espécies e mais de 40 genótipos, sua ocorrência geográfica é mundial e afeta, principalmente, o trato gastrointestinal de mamíferos (incluindo o ser humano), aves, répteis, anfíbios e peixes. Atualmente, já são cerca de 150 espécies de animais que podem ser infectados ${ }^{(1,2)}$. A transmissão da criptosporidiose ocorre devido à ingestão de oocistos de Cryptosporidium spp. (Ordem: Eucoccidiorida, Família: Cryptosporidiidae) em água e/ou alimentos contaminados e por meio do contato direto com material fecal de indivíduos infectados que estejam eliminando a forma infectante ${ }^{(3-9)}$.

Os bovinos podem serinfectadoscom pelomenos quatroespécies de Cryptosporidium: Cryptosporidium parvum, Cryptosporidium bovis, Cryptosporidium ryanae (anteriormente identificado com Cryptosporidium genótipo deer-like) e Cryptosporidium andersoni ${ }^{(10-12)}$. Cryptosporidium parvum é considerado a maior causa de diarreia em bezerros recém-nascidos ${ }^{(13)}$. Este genótipo também pode infectar outros mamíferos incluindo o ser humano, adquirindo potencial zoonótico. Em bovinos, a duração dos sintomas da criptosporidiose depende de inúmeros fatores, incluindo os níveis de contaminação ambiental, a virulência e a infectividade da amostra, a suscetibilidade do hospedeiro e, por fim, a idade à primeira infecção ${ }^{(14)}$.

O objetivo desta pesquisa foi verificar a ocorrência de oocistos de Cryptosporidium spp. em amostras fecais de bezerros (machos e fêmeas) do nascimento até 3 meses de idade, por meio das técnicas de coloração Ziehl-Neelsen modificado e Safranina modificada.

\section{Material e Métodos}

O estudo foi conduzido em cinco pequenas propriedades de criação de bovinos, com aptidão leiteira, localizadas no Município de Uberlândia-Minas Gerais. A escolha foi aleatória, não importando o tamanho e padrão tecnificado. As propriedades apresentavam condições de manejo semelhante, incluindo o sistema de duas ordenhas diárias. Os bezerros eram mantidos em piquetes coletivos, ou seja, sem divisão por faixa etária e bebedouros de uso comum. O período das coletas foi do mês de abril a julho de 2013 .

As amostras de fezes foram obtidas de 152 bezerros do nascimento até 3 meses de idade, a coleta foi realizada diretamente da ampola retal, utilizando-se sacos plásticos descartáveis. Estes foram identificados individualmente e transportados em caixa de isopor com gelo reciclável até a Universidade Federal de Uberlândia - Laboratório de Patologia Clínica Veterinária onde as amostras foram processadas no mesmo dia.

Foram separadas aproximadamente $3 \mathrm{~g}$ de cada amostra fecal, as quais foram diluídas em $10 \mathrm{~mL}$ de água deionizada. Esta solução foi homogeneizada com auxílio do bastão de vidro, em seguida, verteuse em tubo Falcon com capacidade de $15 \mathrm{~mL}$, o material foi centrifugado a 3600 g por 10 minutos. $\mathrm{O}$ sobrenadante foi descartado e a partir do sedimento foram feitos dois esfregaços finos em lâminas de vidro. Uma lâmina foi corada pela técnica de Ziehl-Neelsen modificado ${ }^{(15)}$ e a outra pela técnica da Safranina modificada ${ }^{(16)}$. As lâminas foram observadas ao microscópio óptico em toda sua extensão 
com aumento 1000x em objetiva de imersão. Para a comparação das técnicas de coloração e o sexo, utilizou-se teste não paramétrico (binomial para duas proporções), com nível de significância de 5\% (Programa Bioestat - versão 5.3).

O protocolo foi aprovado pela Comissão de Ética na Utilização de Animais (CEUA/UFU) - registro $\mathrm{n}^{\circ} 116 / 12$.

\section{Resultados}

Das 152 amostras fecais analisadas, 12,5\% (19/152) foram positivas na técnica Ziehl-Neelsen modificado e $7,8 \%(12 / 152)$ na técnica da Safranina modificada, sendo que cinco amostras $(3,2 \%)$ foram positivas em ambas as técnicas de coloração (Tabela 1).

A prevalência geral de Cryptosporidium spp. neste estudo foi de 17,1\% (26/152), independente de a amostra ter sido positiva em uma ou em ambas as técnicas. Quanto ao sexo, não foi verificado diferença estatística (Tabela 2).

Tabela 1: Prevalência de Cryptosporidium spp. em bezerros de 0 a 3 meses, analisadas pelas técnicas de Ziehl-Neelsen modificado e Safranina modificada em animais procedentes de cinco propriedades no município de Uberlândia, MG

\begin{tabular}{llll}
\hline & & \multicolumn{3}{c}{ Percentual de positividade } \\
\hline Propriedade & $\mathrm{N}^{\circ}$ amostras & Ziehl-Neelsen modificado & Safranina modificada \\
\hline 01 & 19 & $4(21,0 \%)$ & $0(0,0 \%)$ \\
02 & $22^{*}$ & $6(27,0 \%)$ & $4(18,0 \%)$ \\
03 & $52^{*}$ & $4(7,6 \%)$ & $2(3,8 \%)$ \\
04 & 28 & $1(3,5 \%)$ & $1(3.5 \%)$ \\
05 & $31^{* *}$ & $11(35,4 \%)$ & $5(16,1 \%)$ \\
\hline Total & 152 & $26(17,1 \%)$ & $12(7,8 \%)$ \\
\hline
\end{tabular}

* Uma amostra positiva nas duas técnicas

** Três amostras positivas nas duas técnicas

Tabela 2: Frequência de bezerros positivos para Cryptosporidium spp. quanto ao sexo, oriundos de cinco propriedades no município de Uberlândia, MG

\begin{tabular}{lllll}
\hline Sexo & Examinados (n) & Positivo (n) & $\%$ & $\mathrm{Z}$ (valor) \\
Macho & 75 & 13 & 17,3 & 0,4706 \\
Fêmea & 77 & 13 & 16,8 & 0,4706 \\
\hline Total & 152 & 26 & 17,1 & \\
\hline
\end{tabular}

Estatisticamente significativo $p<0,05$.

Na Figura 1, observam-se oocistos de Cryptosporidium spp. apresentando a coloração púrpura intenso e o fundo da preparação corado em azul pela técnica de Zhiehl-Neelsen modificado. Na Figura 2, o oocisto de Cryptosporidium spp. aparece corado em vermelho-alaranjado sobre um fundo uniforme corado em verde pela técnica da Safranina modificada. 


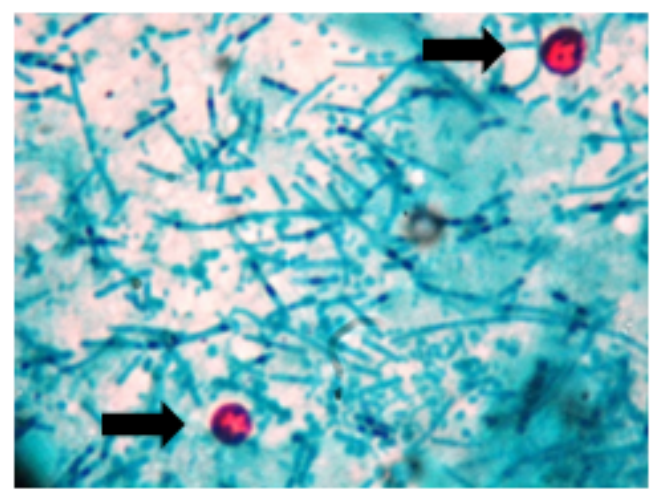

Figura 1: Oocistos de Cryptosporidium spp. (seta) corado pela técnica de Zhiehl-Neelsen modificado. Aumento 1000x em objetiva de imersão. Uberlândia, 2013.

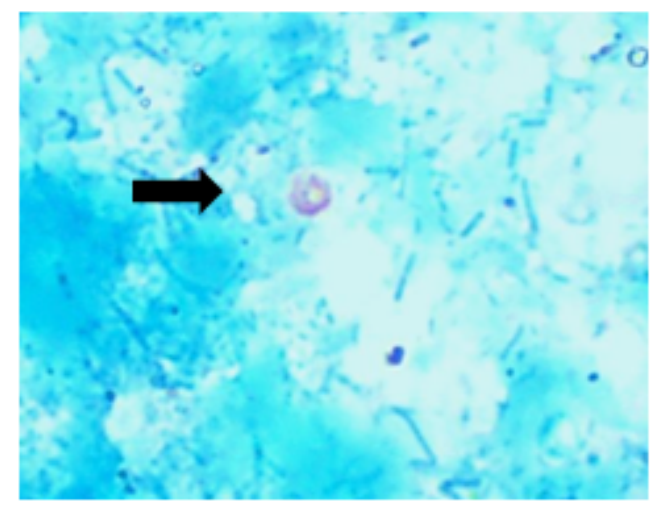

Figura 2: Oocisto de Cryptosporidium spp. (seta) corado pela técnica da Safranina modificada. Aumento $1000 \mathrm{x}$ em objetiva de imersão. Uberlândia, 2013.

\section{Discussão}

No primeiro trabalho sobre a infecção de Cryptosporidium spp. em bovinos, no Brasil, Modolo et al. ${ }^{(17)}$, na região de Botucatu (SP), encontraram $26 \%$ de bezerros menores que 30 dias positivos para esta coccidiose; já nos animais com mais de 30 dias o índice de positividade foi de $23 \%$. A prevalência de Cryptosporidium spp. em bovinos é bastante variada, os índices de positividade encontrados na literatura variam de 0,6 a $82,54 \%$, existindo relato de até $100,0 \%$ de acometimento dos animais ${ }^{(18-20)}$. No presente estudo, a ocorrência de Cryptosporidium spp. em bezerros encontra-se entre os valores observados em estudos conduzidos no Brasil, EUA, Hungria, Alemanha, Irlanda, Irã e Espanha, com a prevalência de $14,0 \%$ a $57,8 \%{ }^{(11,21-25)}$.

Essa variação na prevalência deve-se a inúmeros fatores, dentre eles: delineamento do estudo, idade dos animais, número de amostras por propriedade e metodologia de diagnóstico empregada. Outro fator a ser considerado também é a dificuldade em se identificarem animais assintomáticos ou em período subclínico da infecção, por não eliminarem quantidade detectáveis de oocistos nas fezes ${ }^{(26,27)}$. Quanto às técnicas de coloração utilizadas neste estudo, estas apresentam vantagens e desvantagens e não há um consenso entre os autores. Segundo Rigo et al. ${ }^{(28)}$, a técnica de Ziehl-Neelsen modificado é uma preparação que proporciona uma melhor visualização dos oocistos, sendo também indicada para o diagnóstico das infecções leves, quando o número de oocistos presentes nas fezes é baixo. Segundo Baxby et al. ${ }^{(16)}$, a técnica da Safranina modificada é mais simples e mais rápida que a de Ziehl-Neelsen modificado e apresenta um bom contraste. 
Quanto à faixa etária mais acometida, segundo Pohjola et al. ${ }^{(29)}$, ela se situa dentro da primeira semana de vida. Henriksen e Krough ${ }^{(30)}$ e Bhat et al. ${ }^{(31)}$ afirmaram que a criptosporidiose é mais prevalente em bezerros de 4 a 30 dias. Entretanto, em estudo conduzido por Mtambo et al. ${ }^{(32)}$, a prevalência maior situou-se em bezerros com até três meses. Neste estudo, os animais estão compreendidos nesta faixa etária.

De acordo com Silva Júnior et al. ${ }^{(33)}$, o fornecimento de colostro após as seis primeiras horas de nascimento do bezerro aumenta por volta de quatro vezes o risco de infecção por Cryptosporidium spp., em relação aos animais que receberam o colostro nas primeiras seis horas de vida. Climeni et al. ${ }^{(34)}$ ressaltaram em seu estudo que a qualidade do colostro declina rapidamente após o nascimento do bezerro, bem como a própria capacidade deste animal de absorver as imunoglobulinas que irão conferir imunidade.

Segundo Almeida et al. ${ }^{(35)}$, a presença de reservatório artificial de água disponível para os animais (bebedouro) também é um fator de risco significativo para a presença de Cryptosporidium spp. em fezes de bezerros, pois este pode ser um reservatório de oocistos, que permanecem, por sua vez, viáveis por longos períodos, além de resistirem a processos de filtração e cloração da água. Nas cinco propriedades estudas, os bebedouros eram de uso comum de animais jovens e adultos, sendo um fator importante na contaminação e disseminação da doença entre os animais.

Nas propriedades deste estudo, foi observado que os animais eram mantidos em instalações coletivas e próximos aos currais, o que foi indicado por Silva Júnior et al. ${ }^{(33)}$ como um fator de maior risco para os bezerros, podendo estar expostos a uma maior carga de parasitos no ambiente. Sendo assim, pode-se inferir que o local onde os animais estão alocados são uma importante fonte de disseminação; portanto, a adoção de boas práticas de higiene na criação de bezerros pode reduzir o risco de infecção por Cryptosporidium spp. ${ }^{(36,37)}$.

Quanto ao sexo, este não exerceu influência sobre o índice de infecção por protozoários deste gênero, estando de acordo com Tarazona et al. ${ }^{(38)}$ que afirmaram que a infecção por Cryptosporidium spp. ocorre em indivíduos independentemente do sexo.

\section{Conclusão}

Conclui-se que a infecção por Cryptosporidium spp. está presente nas propriedades avaliadas, porém são necessários mais estudos, para que o risco de infecção seja mensurado adequadamente e medidas profiláticas sejam implementadas.

\section{Referências}

1.Xiao L, Fayer R, Ryan U, Upton SJ. Cryptosporidium taxonomy: recent advances and implications for public health. Clinical Microbiology Reviews.2004,17(1):72-97.

2.Smith HV, Nichols RABC. Cryptosporidium: Detection in water and food. Experimental Parasitology.2010,124(1):61-79.

3.Feng Y, Ortega Y, Ele G, Das P, M Xu, Zhang X, Fayer R, Gatei W, Cama V, Xiao L. Wide geographic distribution of Cryptosporidium bovis and the deer-like genotype in bovines. Veterinary Parasitology.2007,144(1-2):1-9.

4.Langkjaer, RB, Vigre, H, Enemark, HL, Maddox-Hyttel, C. Molecular and phylogenetic characterization of Cryptosporidium and Giardia from pigs and cattle in denmark. Parasitology.2007,137(3):339-350.

5.Coklin T, Uehlinger FD, Farber JM, Barkema HW, O'handley M, Dixon BR. Prevalence and molecular characterization of Cryptosporidium spp. indairy calves from 11 farms in Prince Edward Island, Canada. Veterinary Parasitology.2009,160(3-4):323-326.

6.Boyer DG, Kuczynska E. Prevalence and concentration of Cryptosporidium oocysts in beef cattle paddock soils and forage. Food born Pathogens and Disease.2010,7(8):893-900.

7.Chako CZ, Tyler JW, Schultz LG, Chiguma L, Beerntsen BT. Cryptosporidiosis in people: It's not just about the cows. Journal of Veterinary Internal Medicine.2010,24(1):37-43. 
8.Chalmers RM, Giles M. Zoonotic Cryptosporidiosis in the UK - Challenges for Control. Journal of Applied Microbiology.2010,109(5):1487-1497.

9.Dixon B, Parrington L, Cook A, Pintar k, Pollari F, Farber J. The potential for zoonotic transmission of Giardia duodenalis and Cryptosporidium spp. From beef and dairy cattle in Ontario, Canada. Veterinary Parasitology.2011,175(1-2):20-26.

10.Santín M, Trout JM, Xiao L, Zhou L, Greiner E, Fayer R. Prevalence and age-related variation of cryptosporidium species and genotypes in dairy calves. Veterinary Parasitology.2004,122(2):103-117.

11.Fayer R, Santín M, Xiao L. Cryptosporidium bovis n. sp. (Apicomplexa: Cryptosporidiidae) in cattle (Bos taurus). The Journal of Parasitology.2005,91(3):624-629.

12.Fayer R, Santín M, Trout JM, Greiner E. Prevalence of species and genotypes of Cryptosporidium found in 1- to 2-year-old dairy cattle in eastern United States. Veterinary Parasitology.2006,135:105-112.

13.Wyatt CR, Riggs MW, Fayer R. Cryptosporidiosis in Neonatal Calves. Clínicas Veterinárias da América do Norte: Animal Practice Food.2010,26(1):89-103.

14.Lorenzo MJ, Bem B, Mendez F, Villacorta I, Ares-Mazar ME.Cryptosporidium spp. oocyst antigens recognized by sera from infected asymptomatic adult cattle. Veterinary Parasitology.1995,60(1-2):17-25.

15.Henriksen SA, Pohlenz JFL. Staining of Cryptosporidia by a modified Ziehl- Neelsen technique. Acta Veterinaria Scandinavica.1981,22(3-4):594-596.

16.Baxby D, Blundell N, Hart CA. The development and performance of a simple, sensitive method for the detection of cryptosporidium oocysts in faeces. Journal of Hygiene.1984;93(2):317-323.

17. Modolo JR, Gonçalves RC, Kuchembuck MRG. Ocorrência de Criptosporidiose em Bezerros na Região de Botucatu-SP. Revista Brasileira de Medicina Veterinária.1988,10(1):9-10.

18.Souza JCP, LOPES CWG. Criptosporidiose em bezerros de rebanhos da bacia leiteira Sul-Fluminense, Estado do Rio de Janeiro. Revista Brasileira de Parasitologia Veterinária.1995,4(1):33-36.

19. Cardoso JM, Silveira FL, Araújo AJUS, Carvalho JCC, kanamura HY. Ocorrência de Cryptosporidium spp. em um rebanho bovino leiteiro no município de Caçapava, estado de São Paulo, Brasil. Revista Brasileira de Parasitologia Veterinária.2008;17(supl.1):239-242.

20.Lima RCA, Aquino MCC, Inácio SV, Viol MA, Zucatto AS, Silveira Neto L, Oliveira BCM, Vasconcelos EM, Bresciani KD, Oliveira GP, Costa AJ. Caracterização molecular de Cryptosporidium spp. em bezerros (Bos taurus e Bos indicus) no município de Formiga, Minas Gerais - Brasil. Semina: Ciências Agrárias.2013,34(6) supl.2:3747-3754.

21.Thompson HP, Dooley JSG, Kenny J, Mccooy M, Lowery CJ, Moore JE, Xiao L. Genotypes and subtypes of Cryptosporidium spp. in neonatal calves in northern Ireland. Parasitology Research. 2007,100(3):619-624.

22.Broglia A, Reckinger S, Cacció SM, Nöckler k. Distribution of Cryptosporidium parvum subtypes in calves in Germany. Veterinary Parasitology.2008;154(1-2):8-13.

23.Quílez J, Torres E, Chalmers RM, Hadfield SJ, Del Cacho E, Sánchez-Acedo C. Cryptosporidium genotypes and subtypes in lambs and goat kids in spain. Applied and Environmental Microbiology. 2008,74(19):6026603.

24.keshavarz A, Haghighi A, Athari A, Kazemi B, Abadi A, Mojarad N. Prevalence and molecular characterization of bovine Cryptosporidium in Qazvin province, Iran. Veterinary Parasitology. 2009,160(34):316-318.

25.Paz e Silva FM, Lopes RS, Araújo-Júnior JP. Identification of Cryptosporidium species. Revista Brasileira de Parasitologia Veterinária.2013,22(1):22-28.

26.Ramírez AD, Ramírez-Iglesias LN, Plaza RMG, Román R. Excreción de ooquistes de Cryptosporidium spp. durante el posparto, em vacas mestizas de doble propósito. Revista Cientifica. 2002,12(Supl. 2):614-616.

27.Maddox-Hyttel C, Langkjaer RB, Enemark HL, Vigre H. Cryptosporidium and Giardia in different age groups of Danish cattle and pigs-Occurrence and management associated risk factors. Veterinary Parasitology.2006,141(1-2):48-59.

28.Rigo CR, Franco RM. Comparison entre os Ziehl-Neelsen e Acid-Fast-Trichrome métodos modificados para a pesquisa fecal de Cryptosporidium parvum e Isospora belli. Revista da Sociedade Brasileira de Medicina 
Tropical.2002,35(3).209-214.

29.Pohjola S, Jokipii AMM, Jokipii L. Sporadic Cryptosporidiosis in a rural populations is asymptomatic and associated with contact to cattle. Acta VeterinariaScandinavica.1986,27(1):91-102.

30.Henriksen SA, Krough HV. Bovine Cryptosporidiosis in Denmark 1 - Prevalence, age distribution and seasonal variation. NordiskVeterinaer Medicin.1985,37(1):34-41.

31.Bhat AS, Juyal PD, Singh NK. Coprological investigation on neonatal bovine cryptosporidiosis in Ludhiana, Punjab. Journal of Parasitic Diseases.2013;37(1):114-117.

32.Mtambo MMA, Sebatwale JB, Kambarage DM, Muhairwa AP, Maeda GE, Kusiluka LJM, Kazwala RR. Prevalence of Cryptosporidium spp. oocysts in cattle and wildlife in Morogoro region, Tanzania. Preventive Veterinary Medicine.1997,31(3-4):185-190.

33.Silva Júnior FA, Carvalho AHO, Rocha CMBM, Guimarães AN. Fatores de risco associados à infecção por Cryptosporidium spp. e Giardia duodenalis em bovinos leiteiros na fase de cria e recria na mesorregião do Campo das Vertentes de Minas Gerais. Pesquisa Veterinaria Brasileira.2011, 31(8):690-696.

34.Climeni BSO, Zanatta J, Samaroni M, Monteiro MV. Qualidade do Colostro Bovino. Revista Cientifica Eletrônica de Medicina Veterinária.2008(10):1-5.

35.Almeida AJ, Oliveira FCR, Teixeira C. Risco relativo dainfecção por parasitos do gênero Cryptosporidium em bezerros bovinos no norte do estado do Rio de Janeiro, Brasil. Revista Brasileira de Parasitologia Veterinária.2008;17(supl.1):243-248.

36. Waele V, Speybroeck N, Berkvens D, Mulcahy G, Murphy TM. Control of cryptosporidiosis in neonatal calves: Use of halofuginone lactate in two differentcal frearing systems. Preventive Veterinary Medicine.2010, 96(3-4):143-151.

37.Muhid A, Robertson I, Ng J, Ryan U. Prevalence of and management factors contributing to Cryptosporidium sp. infection in pre-weaned and post-weaned calves in Johor, Malaysia. Experimental Parasitology.2011,127(2):534-538.

38.Tarazona R, Blewett DA, Carmona MD. Cryptosporidium parvum in infection in experimentally infected mice: infection dynamics and effect of immunosuppression. Folia Parasitologica. 1998,45(2):101-107. 